\section{FRI0371 CLINICAL ALGORITHMS FOR THE DIAGNOSIS AND PROGNOSIS OF INTERSTITIAL LUNG DISEASE IN SYSTEMIC SCLEROSIS}

V. $\operatorname{Hax}^{1}$, M. Bredemeier ${ }^{2}$, A.L.D. Moro ${ }^{1}$, T.R. Pavan ${ }^{1}$, M.V. Vieira ${ }^{3}$, E.H. Pitrez ${ }^{3}$, R.M.D.S. Chakr ${ }^{1}$, R.M. Xavier ${ }^{1} .{ }^{1}$ Rheumatology Service, Hospital de Clínicas de Porto Alegre; ${ }^{2}$ Rheumatology Service, Hospital Nossa Senhora da Conceição; ${ }^{3}$ Radiology Service, Hospital de Clínicas de Porto Alegre, Porto Alegre, Brazil

Background: Interstitial lung disease (ILD) is currently the primary cause of death in systemic sclerosis (SSc). Thoracic high-resolution computed tomography (HRCT) is considered the gold standard for diagnosis. Recent studies have proposed several clinical algorithms to predict the diagnosis and prognosis of SSc-ILD.

Objectives: To test the clinical algorithms to predict the presence and prognosis of SSc-ILD, and to evaluate the association of extent of ILD with mortality in a cohort of SSc patients.

Methods: Prospective cohort study, including 177 SSc patients assessed by clinical evaluation, laboratory tests, pulmonary function tests, and HRCT. Three clinical algorithms, combining lung auscultation, chest radiography and \% predicted forced vital capacity (FVC)[1], were applied for the diagnosis of different extents of ILD on HRCT. Univariate and multivariate Cox proportional models were used to analyze the association of algorithms [1,2] and the extent of ILD on HRCT with the risk of death using hazard ratios (HR).

Results: The prevalence of ILD on HRCT was $57.1 \%$ and 79 patients died $(44.6 \%)$ in a median follow-up of 11.1 years. For identification of ILD with extent $\geq 10$ and $\geq 20 \%$ on HRCT, all algorithms presented a high sensitivity $(>89 \%)$ and a very low negative likelihood ratio $(<0.16)$. For prognosis, survival was decreased for all algorithms, especially the algorithm C (HR $3.47,95 \% \mathrm{Cl} 1.62-7.42)$, which identified the presence of ILD based on crackles on lung auscultation, findings on chest X-ray or FVC $<80 \%$. Extensive disease as proposed by Goh and Wells (extent of ILD $>20 \%$ on HRCT or, in indeterminate cases, FVC $<70 \%$ ) had a significantly higher risk of death ( $\mathrm{HR} 3.42,95 \% \mathrm{Cl} 2.12$ to 5.52 ). Survival was not different between patients with extent of 10 or $20 \%$ of ILD on HRCT, and analysis of 10 -year mortality suggested that a threshold of $10 \%$ may also have a good predictive value for mortality. However, there is no clear cutoff above which mortality is sharply increased.

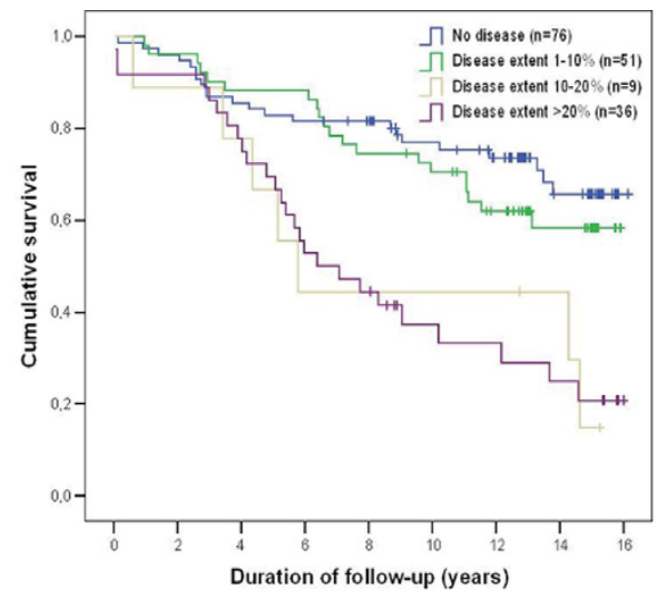

Figure 1. Survival in relation to the extent of lung disease on high-resolution computed tomography ( $p<0.0001$ by log rank test).

Conclusions: Clinical algorithms had a good diagnostic performance for extents of SSc-ILD on HRCT with clinical and prognostic relevance ( $\geq 10$ and $\geq 20 \%)$, and were also strongly related to mortality. Therefore, they probably could be used to obviate the requirement of HRCT in some cases.

References:

[1] Steele R, Hudson M, Lo E, Baron M. Clinical decision rule to predict the presence of interstitial lung disease in systemic sclerosis. Arthritis Care Res (Hoboken) 2012;64:519-24.

[2] Goh NSL, Desai SR, Veeraraghavan S, Hansell DM, Copley SJ, Maher TM, et al. Interstitial lung disease in systemic sclerosis: A simple staging system. Am J Respir Crit Care Med 2008;177:1248-54.

Disclosure of Interest: None declared

DOI: 10.1136/annrheumdis-2017-eular.1448

\section{FRI0372 THE DUCAS: PROPOSAL FOR A DIGITAL ULCER ASSESSMENT SCORE IN SCLERODERMA}

C. Bruni ${ }^{1}$, T. Ngcozana ${ }^{2}$, F. Braschi ${ }^{1}$, S. Guiducci ${ }^{1}$, S. Bellando-Randone ${ }^{1}$, Y.A. Suliman ${ }^{3}$, J. Grotts ${ }^{4}$, C.P. Denton ${ }^{2}$, D.E. Furst ${ }^{5}$, M. Matucci-Cerinic ${ }^{1}$ ${ }^{1}$ Department of Experimental and Clinical Medicine, Division of Rheumatology, University of Florence, Firenze, Italy; ${ }^{2}$ Centre for Rheumatology and Connective Tissue Diseases, UCL Division of Medicine, London, United Kingdom;
${ }^{3}$ Rheumatology and Rehabilitation Department, Assiut University Hospital, Assiut, Egypt; ${ }^{4}$ Department of Biostatistic; ${ }^{5}$ Division of Rheumatology, Department of Medicine, University of California at Los Angeles, Los Angeles, United States

Background: No objective measure is presently available to assess digital ulcer (DU) in SSc patients apart from "healed/non healed" and experience-based clinical judgment.

Objectives: The aim of the current study is to propose a composite DU clinical assessment score (DUCAS) and, to lend it, test its face validity by correlating it with commonly used disease related patient-reported outcomes (PROs) and physician evaluation.

Methods: SSc patients presenting at least one DU and attending the Rheumatology Wound Care Clinic of the Florence University Hospital or the London Royal Free Hospital were enrolled. Patients were assessed with HAQ-DI, Cochin scale, Visual analogic scale (VAS) for DU-related pain (DU_pain, 0-100 mm), patient VAS for global DU status (ptGDU, $0-100 \mathrm{~mm}$ ) and patient global assessment (PtGA, 0-100 mm) as PROs and physician VAS for DU status (phyGDU, 0$100 \mathrm{~mm}$ ). The DUCAS included 7 DU related variables selected by a committee of 8 SSc DU experts - they are outlined in figure 1. Each variable was weighted on a clinical basis and the DUCAS score was the sum of the values for the 7 variables ( $\max =19.5$ ). Spearman's correlation tests were calculated for to examine face validity. A linear regression model with forward and backward stepwise analysis was used to determine the relationship of individual variables with the primary clinical parameter, phyGDU.

Results: 44 SSc patients ( 9 males, mean age $54,3 \pm 15,6$ years, mean disease duration $9,9 \pm 5,8$ years) were enrolled in the study. Mean phyGDU was $44,3 \pm 23 \mathrm{~mm}$, mean ptGDU was $54 \pm 30 \mathrm{~mm}$ (Wilcoxon $p=0.022$, phyGDU VAS vs $p t G D U$ ) and mean DUCAS score was $4,2 \pm 2$. Overall DUCAS showed significant positive correlations with all PROs, but when all the individual clinician and patient's variables were modelled, only the overall DUCAS significantly predicted PhyGDU; after backwards stepwise analysis overall DUCAS and ptGDU best predicted PhyGDU, with an adjusted $\mathrm{R}^{2}=0,437$ and $\mathrm{AIC}=380,3$ (Table 2)

\begin{tabular}{|c|c|c|c|c|c|c|c|c|c|}
\hline \multirow[t]{2}{*}{$\bar{A}$} & \multicolumn{2}{|c|}{$\begin{array}{l}\text { Linear Regression } \\
\text { for DUCAS }\end{array}$} & \multirow[t]{2}{*}{$\mathrm{B}$} & \multicolumn{3}{|c|}{$\begin{array}{l}\text { Linear Model } \\
\text { to PhyGDU }\end{array}$} & \multicolumn{3}{|c|}{$\begin{array}{c}\text { Linear Model to } \\
\text { PhyGDU after } \\
\text { backwards stepwise }\end{array}$} \\
\hline & Spearman Correlation & $p$ & & Estimate & SE & $p$ & Estimate & SE & $p$ \\
\hline PtGA & 0,56 & $<0,001$ & PtGA & 0,011 & 0,199 & 0,955 & & & \\
\hline PtGDU & 0,54 & $<0,001$ & PtGDU & 0,171 & 0,233 & 0,467 & 0,272 & 0,101 & 0,01 \\
\hline DU_Pain & 0,44 & 0,003 & DU_Pain & 0,048 & 0,182 & 0,793 & & & \\
\hline HAQ-DI & 0,44 & 0,003 & $\mathrm{HAQ}-\mathrm{DI}$ & 4,58 & 7,563 & 0,549 & & & \\
\hline COCHIN & 0,51 & $<0,001$ & COCHIN & 0,035 & 0,252 & 0,891 & & & \\
\hline PhyGDU & 0,63 & $<0,001$ & DUCAS & 4,636 & 1,617 & 0,007 & 4,841 & 1,489 & 0,002 \\
\hline
\end{tabular}

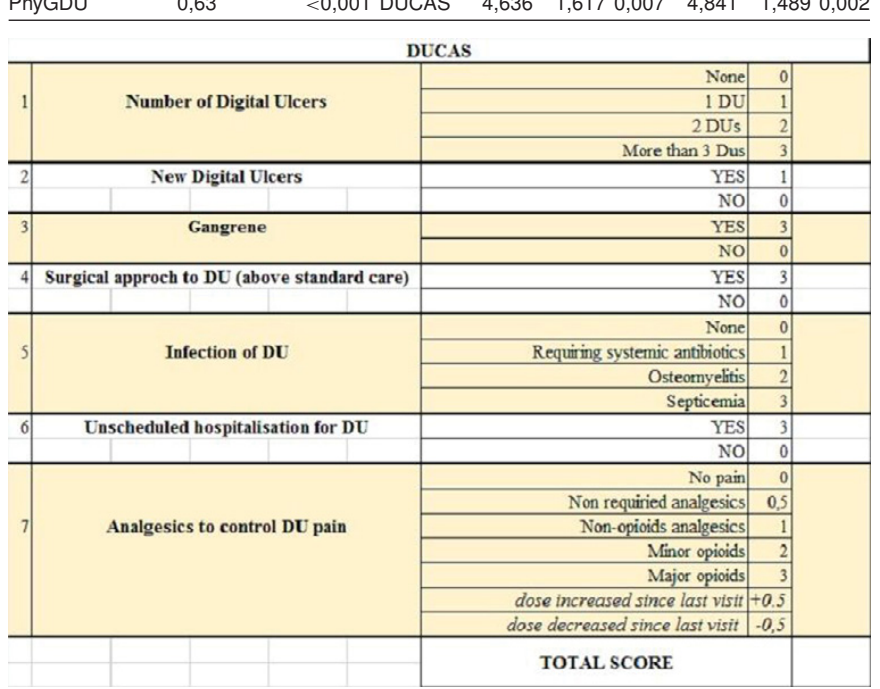

Conclusions: DUCAS is a newly proposed clinical score for SSc related DU which has face validity and which may reflect DU status as judged by SSc experts. Further validation of this score will be undertaken

Acknowledgements: Cosimo Bruni received a EULAR travel bursary to run this project in the UK.

Disclosure of Interest: None declared

DOI: 10.1136/annrheumdis-2017-eular.5735 